\title{
Interpreting the results of searches for gravitational waves from coalescing binaries
}

\author{
Patrick R Brady ${ }^{1}$ and Stephen Fairhurst ${ }^{2,3}$ \\ ${ }^{1}$ University of Wisconsin-Milwaukee, Milwaukee, WI 53201, USA \\ ${ }^{2}$ LIGO_California Institute of Technology, Pasadena, CA 91125, USA \\ ${ }^{3}$ School of Physics and Astronomy, Cardiff University, Cardiff, CF2 3YB, UK
}

Received 15 November 2007, in final form 12 March 2008

Published 29 April 2008

Online at stacks.iop.org/CQG/25/105002

\begin{abstract}
We describe a method to obtain an astrophysical result from the output of a search for gravitational waves from coalescing binaries. Specifically, we introduce a method based on the loudest event statistic to calculate an upper limit or interval on the astrophysical rate of binary coalescence. The calculation depends upon the sensitivity and noise background of the detectors, and a model for the astrophysical distribution of coalescing binaries. There are significant uncertainties in the calculation of the rate due to both astrophysical and instrumental uncertainties as well as errors introduced by using the post-Newtonian waveform to approximate the full signal. We catalog these uncertainties in detail and describe a method for marginalizing over them. Throughout, we provide an example based on the initial LIGO detectors.
\end{abstract}

PACS numbers: $04.30 . \mathrm{Tv}, 04.80 . \mathrm{Nn}$, 95.85.Sz, 07.05.Kf

(Some figures in this article are in colour only in the electronic version)

\section{Introduction}

The first generation of gravitational wave interferometric detectors have achieved, or are approaching, their design sensitivities [1-3]. One of the most promising sources of gravitational waves for these detectors are those emitted during the coalescence of a binary system composed of neutron stars or black holes. The initial detectors will be sensitive to binary neutron star (BNS) coalescences as far away as the Virgo cluster, while for binary black holes $(\mathrm{BBH})$ the reach is as great as $100 \mathrm{Mpc}$. Thus, with a year of data, there is a possibility of detecting gravitational waves from these sources. Even in the absence of a detection, the upper limits which will be placed on the rates of binary coalescences will begin to be astrophysically interesting. Following the initial searches, the detectors will be upgraded to enhanced and advanced configurations where the sensitivity will increase by factors of around two and ten, respectively, over the initial detectors. In this paper, we describe a method which can be 
used for providing an astrophysical interpretation of the results of a search for compact binary coalescence. In the absence of a detection, this will result in an upper limit on the rate of such events. If a signal is observed, then a rate interval will be calculated. The rate limit calculation makes use of the loudest event statistic, first described in [4] and elaborated upon in [5].

The rate of gravitational waves observable at the detectors depends upon the detector sensitivity and also the astrophysical model for the source in question. The standard assumption [6] is that the rate of binary coalescence in a galaxy follows the blue-light luminosity, which depends upon the star formation rate. At cosmological distances, it is appropriate to treat the universe as homogeneous and isotropic. However, for the initial and enhanced gravitationalwave detectors, local anisotropies can have a significant effect. In particular, for initial LIGO and Virgo, a significant fraction of the available luminosity comes from the Virgo cluster [7]. Therefore, it is critically important to generate an accurate distribution of the blue light in the local universe. In [8] a catalog of local galaxies has been constructed precisely for this purpose. In this paper, we describe in detail how this galaxy catalog can be used to calculate the total luminosity to which a gravitational-wave detector is sensitive. In addition to the galaxy distribution, this is dependent upon evaluating the ability of a search to detect a binary with given parameters. We describe how this search efficiency can be calculated and folded together with the galaxy distribution to obtain a total luminosity for a given search.

The amplitude of the gravitational-wave signal emitted by a coalescing binary is dependent upon the component masses of the binary. Thus, a rate calculation (upper limit or interval) obtained from a search of gravitational-wave data will be sensitive to the astrophysical model for the distribution of the component masses of the binary. Although several neutron stars in binaries, and many isolated neutron stars, have been observed as pulsars, there is still significant uncertainty in the mass distribution [9]. Furthermore, given the lack of observed neutron star-black hole or binary black hole systems, the distribution of component masses of these systems is highly uncertain. With this in mind, we describe a simple method whereby mass-dependent rates can be obtained.

In order to claim the detection of gravitational waves, it is vitally important to have a good understanding of the distribution of events which are due to instrumental noise. The output from a search for gravitational-wave transients is a list of candidate events which survive all thresholds applied during the search. These candidate events can contain both background, noise events as well as true gravitational-wave signals. Without a good understanding of the noise background, it will be impossible to determine that an event is due to a gravitational-wave signal. We shall see in detail later that a good estimate of the noise background is equally important for calculating rate limits or intervals.

There are numerous uncertainties affecting our understanding of the astrophysics, instrumental sensitivities and background estimates required in interpreting the results. The uncertainties in the galaxy distribution are detailed in [8]. While the sky position of nearby galaxies is well known, their distance from the Earth and blue-light luminosity is measured less accurately. The search for gravitational waves from coalescing binaries makes use of waveform templates calculated using the post-Newtonian approximation to general relativity. This will lead to some discrepancy between the waveform used in the search and true physical waveform, particularly close to coalescence. In addition, the spin of the binaries is neglected in current searches [10]. However, neutron stars and black holes occurring in binary systems are expected to be spinning. The background of noise events is estimated by time shifting the data from each instrument relative to the others. This provides a reasonable, but not perfect estimate of the background rate. Furthermore, instrumental calibration uncertainties will affect the reported sensitivity of the instrument. All of these will have an effect on 
the calculated rates. We provide a detailed discussion of these systematic uncertainties and describe a method by which these can be incorporated into the final rate statements.

The layout of the paper is as follows. First, in section 2 we briefly review the techniques used in searching for gravitational waves from coalescing binaries. In section 3 we discuss the loudest event statistic, and demonstrate its application to a search for binary inspiral. In section 4 we describe the various systematic effects which can effect the rate upper limit, and describe a method for marginalizing over these uncertainties. Finally, in section 5 we summarize the results.

Throughout the paper, the methods discussed are applied to an illustrative example. This example involves simulated results from two detectors operating at the initial LIGO sensitivity for 1 year. More concretely we choose instruments with a binary neutron star horizon distance (the distance at which an optimally oriented and located system would produce a signal-tonoise ratio (SNR) of 8 in a single detector) of $35 \mathrm{Mpc}$. This is consistent with sensitivities achieved by the Hanford and Livingston $4 \mathrm{~km}$ detectors (H1 and L1 respectively) during the latter parts of the S5 science run. Furthermore, we assume that the detectors and search are such that the SNR threshold can be set at 5.5 and noise background will produce an expected rate of one event per year at a combined SNR of 10, or equivalently about 7 in each instrument. This value is somewhat greater than the combined SNR of 8 often assumed (see, for example, [11]) and reflects a realistic value given the non-stationarity of the initial detectors. Although the example presented here involves simulated results, the methods are easily generalized to searches of real data. Indeed, the reported upper limits from searching the data from the third and fourth LIGO science runs [10] were obtained using the methods described here.

\section{Details of inspiral searches}

The gravitational waveform emitted by a coalescing binary can be calculated to high accuracy within the post-Newtonian expansion of general relativity. In section 2.1, we sketch the details of the inspiral waveform, paying particular attention to those quantities which have a direct impact on the detectability of the waveform at a given detector. Then, in section 2.2 , we describe the matched-filter search method which is used in searching for the waveform. We finish with a brief description of the extension to multi-detector searches.

\subsection{The waveform}

The gravitational-wave strain incident on an interferometer is given by

$$
h(t)=F_{+}(\theta, \phi, \psi) h_{+}(t)+F_{\times}(\theta, \phi, \psi) h_{\times}(t),
$$

where $F_{+}$and $F_{\times}$are the detector response functions and $h_{+}, h_{\times}$are the two polarizations of the gravitational wave. The detector response functions depend upon the sky location $(\theta, \phi)$ and polarization angle $\psi$ of the source relative to the detector according to [12]

$$
\begin{aligned}
& F_{+}=-\frac{1}{2}\left(1+\cos ^{2} \theta\right) \cos 2 \phi \cos 2 \psi-\cos \theta \sin 2 \phi \sin 2 \psi, \\
& F_{\times}=\frac{1}{2}\left(1+\cos ^{2} \theta\right) \cos 2 \phi \sin 2 \psi-\cos \theta \sin 2 \phi \cos 2 \psi .
\end{aligned}
$$

For a low-mass binary coalescence, the portion of the waveform which is available to the LIGO and Virgo detectors can be calculated using the restricted post-Newtonian expansion to a high accuracy [13-23]. For restricted waveforms, only the leading order term in the amplitude is used, while post-Newtonian corrections to the phase are retained. The waveform depends upon the masses and spins of the binary components, the orientation and distance of the binary relative to the detector. In this paper, we restrict attention to searches which make 
use of waveforms appropriate for binaries without spin. In section 4.2 we estimate the effect of using non-spinning templates in the search for spinning waveforms.

The post-Newtonian expression for the binary inspiral waveform can be substituted into equation (1) to obtain an alternative expression for the gravitational-wave strain at the detector $[24,25]$. In this form, the restricted waveform is expressed in terms of the two phases- $h_{c}$, the cosine phase, and $h_{s}$, the sine phase-of the chirp waveform, and an overall amplitude and phase factor. By doing so, there is a clear split between the overall scaling factors which depend upon the distance, sky location and orientation of the binary and the mass-dependent time evolution of the waveform. The waveform is written as

$$
h(t)=\frac{1 \mathrm{Mpc}}{D_{\text {eff }}}\left[h_{c}(t) \cos \Phi+h_{s}(t) \sin \Phi\right]
$$

where the amplitude factor $D_{\text {eff }}$ is known as the effective distance to the binary and $\Phi$ is the coalescence phase as observed at the detector. Both the effective distance and coalescence phase are determined by the location and orientation of the binary system relative to the detector. More specifically, the effective distance is defined as [26]

$$
D_{\text {eff }}=\frac{r}{\sqrt{F_{+}^{2}\left(1+\cos ^{2} \iota\right)^{2} / 4+F_{\times}^{2} \cos ^{2} \iota}},
$$

where $r$ is the physical distance to the binary, $\iota$ is the inclination angle of the binary system and $F_{+}, F_{\times}$are given in (2). Similarly, the phase angle $\Phi$ is dependent upon the sky location, polarization, inclination and also the coalescence phase $\phi_{o}$ of the binary.

Define the Fourier transform of $h(t)$ by

$$
\tilde{h}(f)=\int_{-\infty}^{\infty} h(t) \mathrm{e}^{-2 \pi \mathrm{i} f t} \mathrm{~d} t .
$$

The sine and cosine phases of the binary inspiral waveform are dependent upon the component masses. In the frequency domain, they are obtained using the stationary phase approximation to the post-Newtonian expansion [27]. In this approximation, $\tilde{h}_{s}(f)=\mathrm{i} \tilde{h}_{c}(f)$, and

$$
\tilde{h}_{c}(f)=\mathcal{N}\left[\frac{G \mathcal{M}}{c^{3}}\right]^{5 / 6} \Theta\left(f-f_{\max }\right) f^{-7 / 6} \mathrm{e}^{\mathrm{i} \Psi(f ; \mathcal{M}, \eta)},
$$

where $\mathcal{N}$ is an overall (known) scaling, $\Theta$ is the Heaviside step function and we have introduced the chirp mass $\mathcal{M}=M \eta^{3 / 5}$, where $M=\left(m_{1}+m_{2}\right)$ is the total mass and $\eta=m_{1} m_{2} / M^{2}$ is the symmetric mass ratio. The phase evolution is governed by $\Psi(f ; \mathcal{M}, \eta)$ which also depends upon the masses of the binary system. The waveform terminates at a frequency $f_{\max }$. Physically, the post-Newtonian waveform is expected to break down around the frequency where the evolution changes from a slow inspiral to a rapid merger. A reasonable estimate of this frequency is given by the innermost stable circular orbit (ISCO) of the Schwarzschild spacetime with the same total mass $M$,

$$
f_{\text {isco }}=\frac{2.8 M_{\odot}}{M} 1600 \mathrm{~Hz} \text {. }
$$

In principle, the waveform observed at the detector for a non-spinning binary system depends upon eight parameters: the masses of the two binary components, the physical distance $r$, the sky location and polarization $(\theta, \phi, \psi)$, the inclination angle $\iota$ and the coalescence phase $\phi_{o}$. However, it is clear from equation (6) that the six quantities describing the location and orientation of the binary affect the strain observed at a single detector only in the combinations $D_{\text {eff }}$ and $\Phi$. Furthermore, the ability to distinguish a gravitational wave from a coalescing binary above the background noise is independent of the coalescence phase at the detector. Finally, it is only the chirp mass which affects the amplitude of the waveform (the mass ratio 
$\eta$ does affect the phase evolution). Therefore, the detection efficiency will depend primarily upon the effective distance $D_{\text {eff }}$ and the chirp mass $\mathcal{M}$. This observation will be used to greatly simplify the rate calculation.

\subsection{Inspiral search method}

Since the inspiral waveform is well known, the standard matched filtering technique is used to distinguish signal from noise in a single detector [28]. Here, we provide a very brief review of the search implementation, further details are available in [24, 25]. The gravitational waveform from a coalescing binary given in equation (6) depends upon the masses, effective distance and coalescence phase of the binary. The two mass dimensions are searched by covering the mass space with a template bank which guarantees that for any signal there is a good overlap between the waveform and the closest template [29]. As discussed below, the coalescence phase is analytically maximized over in the matched filtering process and the distance is measured.

The output of a detector is

$$
s(t)=n(t)+h(t),
$$

where $n(t)$ is the instrumental noise and $h(t)$ is some, possibly absent, signal. The sensitivity of the instrument is characterized by the (one-sided) power spectrum $S(f)$, given as

$$
\left\langle\tilde{n}(f) \tilde{n}^{*}\left(f^{\prime}\right)\right\rangle=\frac{1}{2} \delta\left(f-f^{\prime}\right) S(|f|),
$$

where the tilde represents the Fourier transform, and the angle brackets denote the expectation value over the noise. In order to construct the matched filter, we introduce an inner product

$$
(a \mid b):=4 \operatorname{Re} \int_{0}^{\infty} \mathrm{d} f \frac{\tilde{a}(f) \tilde{b}^{*}(f)}{S(|f|)} .
$$

The sensitivity of the detector to a given signal is characterized by

$$
\sigma^{2}=\left(h_{c} \mid h_{c}\right),
$$

where $\sigma^{2}$ depends upon the noise curve of the instrument as well as the masses of the binary components (recall that $h_{c}$ is the waveform at an effective distance of $1 \mathrm{Mpc}$ ). The SNR is defined as

$$
\rho^{2}=\frac{\left(s \mid h_{c}\right)^{2}+\left(s \mid h_{s}\right)^{2}}{\sigma^{2}} .
$$

The analytic maximization over the unknown phase angle has already been performed by including the outputs from the two orthogonal filters, $h_{c}$ and $h_{s}$, while the amplitude of the signal determines the value of the SNR.

In the absence of a signal, the SNR squared is $\chi^{2}$ distributed with two degrees of freedomone for each of the filters. Thus for a single trial, the probability of obtaining an SNR greater than $\rho_{*}$ is

$$
P\left(\rho^{2}>\rho_{*}^{2}\right)=\mathrm{e}^{-\rho_{*}^{2} / 2} .
$$

If the detector output contains a signal $h(t)$, we characterize its amplitude by

$$
\rho_{h}^{2}:=(h \mid h)=\frac{\sigma^{2}}{D_{\text {eff }}^{2}} .
$$

In this case, the SNR squared is distributed as a non-central $\chi^{2}$ distribution with two degrees of freedom and a non-centrality parameter $\rho_{h}^{2}$. Thus, the expected SNR squared is $\rho_{h}^{2}+2$ while the variance is $4\left(\rho_{h}^{2}+1\right)$. For SNRs well above unity, the expected SNR is close to $\rho_{h}$. 
In the course of a gravitational wave search, we calculate the SNR for every mass template in the bank and for every time point. This is used to construct a list of times and associated mass parameters at which the SNR exceeds some pre-determined threshold. These candidate events may be due to instrumental noise or gravitational waves. In searching a year of data over a wide range of masses, we obtain a background of events due to noise with a distribution consistent with (13). In addition, the data from the detectors contain non-stationarities which also produce events with high SNR. The ability to reduce this background of non-gravitational wave induced events affects the sensitivity of the search. There are many techniques employed to achieve this [30]. The most powerful tool is a consistency test between detectors-the arrival time of the signal should be consistent, within the light travel time between the sites, and the mass parameters should agree within the search accuracy. In addition, signal consistency tests, such as the $\chi^{2}$ [31] and $r^{2}$ tests [32] are utilized, and an 'effective SNR', constructed using the SNR and $\chi^{2}$ information, is used to distinguish signal from noise [10,30]. After applying these tests, the typical loudest surviving background events for BNS searches have a combined $\operatorname{SNR} \rho=\sqrt{\rho_{1}^{2}+\rho_{2}^{2}}$ of around 10. For higher mass systems, the waveforms spend a shorter time in the sensitive band. Consequently, it is more difficult to distinguish them from noise non-stationarities whence the background extends to higher SNR.

\section{Rate calculations for inspiral searches}

Let us assume that a search for coalescing binaries has been performed on a stretch of data from gravitational-wave detectors. We would like to use the search results to make a statement about the rate of binary coalescences in the universe. This can be done by making use of the loudest event statistic, as described in $[4,5]$. The result will depend upon the astrophysical model for the distribution of binary coalescences. To proceed, we make the simple, yet astrophysically reasonable [6], assumption that binary coalescences occur only in galaxies and furthermore the rate of binary coalescence in a given galaxy is directly proportional to the blue-light luminosity of that galaxy. This assumption is justified by the fact that both the star formation rate and supernova rate are proportional to the blue-light luminosity. The result from a search for coalescing binaries, in the absence of a detection, will be a bound on the rate

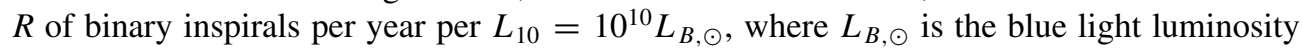
of the sun. Recent papers have suggested that due to the large delay between formation and coalescence for binaries, this simple assumption will underestimate the contribution from elliptical galaxies, particularly for $\mathrm{BBH}$ rates [33]. The formalism presented below can be modified in a straightforward manner to take this into account. The only requirement is that we have a model for the distribution of coalescing binaries in the universe.

The loudest event statistic makes use of the fact that no events with an SNR greater than that of the loudest event $\rho_{m}$ occurred in the search. For an inspiral rate $R$, the probability of there being no gravitational-wave signals with SNR greater than $\rho$ is ${ }^{4}$

$$
P_{F}(\rho)=\mathrm{e}^{-R \mathcal{C}_{L}(\rho) T},
$$

where $\mathcal{C}_{L}(\rho)$ is the total luminosity to which the search is sensitive and $T$ is the duration of the search. Similarly, the probability of obtaining zero accidental noise (or background) events with an SNR greater than $\rho$ is denoted as $P_{B}(\rho)$. Therefore, the overall probability of obtaining no events with SNR greater than $\rho$ is

$$
P(\rho \mid B, R, T)=P_{B}(\rho) \mathrm{e}^{-R \mathcal{C}_{L}(\rho) T} .
$$

4 The notation $F$ is used to denote 'foreground' or gravitational-wave events, in contrast to background $B$ events associated with instrumental noise. 
Given that no event was observed with SNR greater than that of the loudest event $\rho_{m}$, a straightforward application of Bayes' theorem leads to the posterior probability distribution for the rate $p\left(R \mid \rho_{m}, T, B\right)$ which depends upon the loudest event, amount of time searched and the accidental rate (encoded as 'B') [5]. In addition, it will depend upon the prior probability distribution for the rate, denoted as $p(R)$. In the absence of previous knowledge of the rate, a uniform prior $p(R)=$ const is appropriate, while if a previous search has been performed, the posterior of that search is naturally used as the prior for the next search. The expression for the posterior distribution is

$$
p\left(R \mid \rho_{m}, T, B\right) \propto p(R)\left[\frac{1+\Lambda R \mathcal{C}_{L}\left(\rho_{m}\right) T}{1+\Lambda}\right] \mathrm{e}^{-R \mathcal{C}_{L}\left(\rho_{m}\right) T} .
$$

Here, $\Lambda$ is a measure of the relative probability that the loudest event is a due to a gravitational wave, rather than from instrumental noise. The expression for $\Lambda$ is

$$
\Lambda=\frac{\left|\mathcal{C}_{L}^{\prime}\left(\rho_{m}\right)\right|}{P_{B}^{\prime}\left(\rho_{m}\right)}\left[\frac{\mathcal{C}_{L}\left(\rho_{m}\right)}{P_{B}\left(\rho_{m}\right)}\right]^{-1}
$$

where the derivatives are taken with respect to $\rho$. The quantity $\Lambda$ depends upon the population through the total luminosity $\mathcal{C}_{L}\left(\rho_{m}\right)$ and its derivative with respect to $\operatorname{SNR}, \mathcal{C}_{L}^{\prime}\left(\rho_{m}\right)$. Similarly it depends upon the background distribution of noise events through $P_{B}\left(\rho_{m}\right)$ and its derivative. For practical calculational purposes, it is often useful to write

$$
\Lambda=\frac{n_{F}}{n_{B}} \quad \text { where } \quad n_{F}=\frac{\left|\mathcal{C}_{L}^{\prime}\left(\rho_{m}\right)\right|}{\mathcal{C}_{L}\left(\rho_{m}\right)}, \quad n_{B}=\frac{P_{B}^{\prime}\left(\rho_{m}\right)}{P_{B}\left(\rho_{m}\right)} .
$$

By doing this, the dependence upon the estimated background is confined to $n_{B}$ while the quantity $n_{F}$ depends only upon the astrophysical population model.

The posterior distribution obtained in equation (17) can be used to calculate a Bayesian upper limit on the rate. The upper limit $R_{*}$ for a given confidence level $(\alpha)$ is obtained by evaluating

$$
\alpha=\int_{0}^{R_{*}} p\left(R \mid \rho_{m}, T, B\right)
$$

Assuming a uniform prior on the rate, the upper limit is given by

$$
1-\alpha=\mathrm{e}^{-R_{*} T \mathcal{C}_{L}\left(\rho_{m}\right)}\left[1+\left(\frac{\Lambda}{1+\Lambda}\right) R_{*} T \mathcal{C}_{L}\left(\rho_{m}\right)\right] .
$$

In the case where the loudest event candidate is most likely due to the background $\Lambda \rightarrow 0$ and the upper limit becomes $R_{90 \%}=2.3 /\left[T \mathcal{C}_{L}\left(\rho_{m}\right)\right]$.

In the limit that $\Lambda \rightarrow \infty$, the event is almost definitely due to a gravitational wave rather than from the noise background. In this limit, the probability distribution in equation (17) is peaked away from zero, whence it makes sense to construct a rate interval (as described in [5]) rather than a rate upper limit. This is done by performing the integral in (20) from $R_{\min }$ to $R_{\max }$. Alternatively, one could choose to still place an upper limit, in which case $R_{90 \%}=3.9 /\left[T \mathcal{C}_{L}\left(\rho_{m}\right)\right]$.

In the remainder of this section, we discuss how the quantities $\Lambda$ and $\mathcal{C}_{L}$ can be calculated. In section 3.1, we describe the estimation of the noise background and the evaluation of $n_{B}$. Then, in section 3.2, we describe the calculation of the luminosity $\mathcal{C}_{L}$ and consequently $n_{F}$. Finally, in section 3.3, we combine these results to obtain an expression for the upper limit. 


\subsection{Estimating the noise background}

In order to calculate an upper limit, we require an estimate of the background of events due to noise in the detectors. For perfectly Gaussian, stationary detectors, this can be calculated theoretically using the known distribution for the SNR. However, real detectors suffer from non-stationarities and time varying sensitivity. Thus, for a search involving several detectors, the background is most readily estimated by time shifting the data from the detectors relative to one another, and then searching for events which are coincident in time and mass between the detectors. If the time shifts are greater than the light travel time and length of the signal, then the time shifted coincidences cannot be due to gravitational waves, and are therefore expected to give a good estimate of the background. In practice, time shifting by multiples of $5 \mathrm{~s}$ [10] has been found to be sufficient. Even though some signals last longer than this, the inner product (10) between two signals displaced in time by $5 \mathrm{~s}$ is significantly smaller than the SNR of the signals.

Each time shift will have a loudest event, which we assume to be drawn from the actual background distribution for the loudest event, $p_{B}(\rho):=P_{B}^{\prime}(\rho)$. Therefore, by performing a large number of time shifts, we obtain a good sampling of $p_{B}(\rho)$ from which it is straightforward to obtain the cumulative distribution $P_{B}(\rho)$ and $n_{B}=p_{B}\left(\rho_{m}\right) / P_{B}\left(\rho_{m}\right)$.

As an example, consider a pair of detectors whose noise output is Gaussian and stationary. In this case, the noise background for a single detector is Poisson distributed, with rate of events louder than $\rho_{i}$ being given by

$$
\mu\left(\rho_{i}\right)=C \mathrm{e}^{-\rho_{i}^{2} / 2}
$$

where $C$ depends upon the number of trials. With two detectors, the combined SNR is given by $\rho^{2}=\rho_{1}^{2}+\rho_{2}^{2}$. Furthermore, it is necessary to impose a single detector threshold on the SNR, denoted as $\rho_{T}$. In this case, the Poisson rate of background events is

$$
\mu(\rho)=C\left(1+\rho^{2} / 2-\rho_{T}^{2}\right) \mathrm{e}^{-\rho^{2} / 2} .
$$

The constant $C$ can be determined from the expected loudest event. Given the Poisson rate $\mu(\rho)$, it is straightforward to calculate the distributions of $P_{B}, p_{B}$ and $n_{B}$,

$P_{B}(\rho)=\mathrm{e}^{-\mu(\rho)}, \quad p_{B}(\rho)=\left|\mu^{\prime}(\rho)\right| \mathrm{e}^{-\mu(\rho)} \quad$ and $\quad n_{B}=\left|\mu^{\prime}(\rho)\right|$.

Given the background of (23), at the expected loudest event, $n_{B}\left(\rho_{m}\right) \approx \rho_{m}$.

In our example, we choose $\rho_{T}=5.5$ and select $C$ such that the expected loudest event has an SNR of 10, i.e. $\mu(10)=1$, equivalent to an SNR of about 7 in each detector. For this search, we simulate 100 time shifts and obtain the loudest event for each. The distributions are plotted in figure 1. The features in these plots are due to the finite number of time-shifts performed, which lead to uncertainties in the reconstruction of the distributions. In addition, we simulate the output of the search, and obtain a loudest event with $\rho_{m}=9.95$ which yields values of $p_{B}=2.7, P_{B}=0.25$ and $n_{B}=10.9$.

\subsection{Calculating the foreground}

The upper limit derived from a search depends upon the total luminosity $\mathcal{C}_{L}$ to which a search is sensitive. This must be evaluated at the SNR of the loudest event $\mathcal{C}_{L}\left(\rho_{m}\right)$. In section 2 , we have shown that the strength of the gravitational-wave signal at a detector depends primarily upon the effective distance $D_{\text {eff }}$ and chirp mass $\mathcal{M}$ of the signal. In addition, the measured SNR of signal with a given $D_{\text {eff }}$ and $\mathcal{M}$ will fluctuate depending upon the noise in the detectors at the time. Thus, for each value of $D_{\text {eff }}$ and $\mathcal{M}$, we can calculate the probability that the signal is observed with an SNR greater than $\rho$. This quantity is known as the efficiency, 

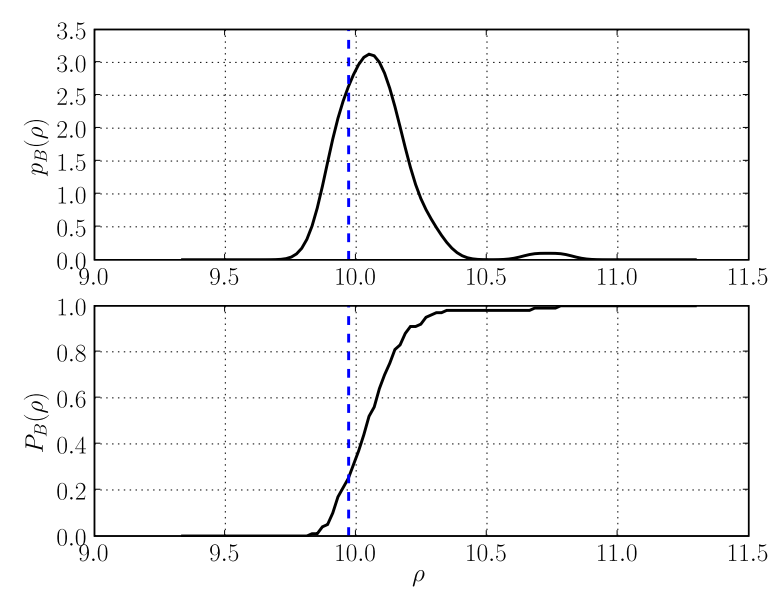

Figure 1. The distribution of the SNR of the loudest event. These distributions were obtained by generating 100 time-shift loudest events from the distributions in equation (24) with an event rate $\mu(\rho)$ given by equation (23), with the normalization constant chosen so that the expected rate is unity at an SNR of 10 . The upper plot shows the probability distribution $p_{B}(\rho)$, while the lower plot shows the cumulative distribution $P_{B}(\rho)$. The distribution is, as expected, peaked close to an SNR of 10. The small bump at an SNR of around 10.7 is due to one of the time shift events being louder than average and illustrates the difficulty in determining a probability distribution from 100 measurements only. The cumulative probability tends to zero at small SNR - it is almost guaranteed that there will be an event louder - and unity at large SNR - it is very unlikely to have an event louder than this. The dashed line in both plots shows the simulated result with an SNR of 9.95 .

$\epsilon\left(\rho, D_{\text {eff }}, \mathcal{M}\right)$. Since the sensitivity of a detector varies over the course of a search, the efficiency is measured by performing Monte Carlo simulations. For a search involving several detectors, the efficiency will depend upon the effective distance to the source from all detectors, which we denote in bold as $\mathbf{D}_{\text {eff }}$. The efficiency calculation is discussed in section 3.2.1.

To calculate the total luminosity, we also require a measure of the blue-light luminosity $L_{B}\left(D_{\text {eff }}, \mathcal{M}\right)$ as a function of $D_{\text {eff }}$ and $\mathcal{M}$. This is calculated from the known luminosity density in the universe. For the initial LIGO detectors - with sensitivity to binary neutron star coalescences up to $40 \mathrm{Mpc}$ - it is necessary to take into account the inhomogeneity of the local universe. This requires the construction of a catalog of nearby galaxies (see [8] for details on how this is constructed). Armed with a galaxy catalog and a mass distribution for the binaries, the method of calculating $L_{B}$ is described in section 3.2.2. by

Finally, given the efficiency and luminosity functions, the cumulative luminosity is given

$$
\mathcal{C}_{L}(\rho)=\int \mathrm{d} \mathbf{D}_{\text {eff }} d \mathcal{M} \epsilon\left(\rho, \mathbf{D}_{\text {eff }}, \mathcal{M}\right) L_{B}\left(\mathbf{D}_{\text {eff }}, \mathcal{M}\right)
$$

Details of this calculation are given in section 3.2.3.

3.2.1. Efficiency. The efficiency of a search is evaluated by adding simulated inspiral signals to the data stream and evaluating the probability that signals with a given set of parameters are detected with SNR greater than $\rho_{m}$. By performing a host of injections, it is possible to evaluate the efficiency as a function of both the chirp mass and effective distance. 


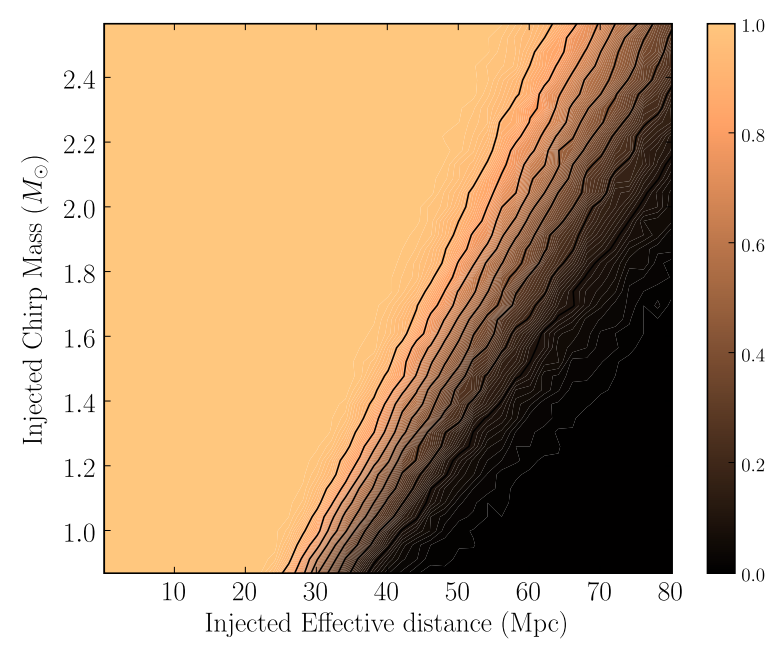

Figure 2. The efficiency of the initial LIGO detectors to coalescing binaries as a function of the effective distance and chirp mass of the source. The efficiency curve is evaluated at an SNR of 7 in the detector. For a fixed chirp mass, the efficiency is unity at small distances and decreases to zero as the distance increases, as expected from equation (14). The chirp mass dependence follows immediately from equation (6).

Figure 2 shows the simulated efficiency of the initial LIGO $4 \mathrm{~km}$ detectors, at an SNR of 7 , as a function of the binary's effective distance and chirp mass. The shape of the efficiency curve for a given chirp mass follows directly from equation (14) and the surrounding discussion: the efficiency is unity at small distance and decreases to zero as the distance increases. The shape of the curve is determined by the observed SNR distribution due to noise fluctuations. The distance to which events are detectable increases with the chirp mass. This follows immediately from equation (6) for the inspiral waveform, from which we see that the amplitude is proportional to $\mathcal{M}^{5 / 6} / D_{\text {eff }}$. For binaries with a total mass less than $10 M_{\odot}$, the inspiral stage of the evolution will sweep right across the sensitive band of the detector. Therefore, for low-mass signals, the detectability of a signal at a given detector will be dependent only on the chirp distance $D_{\mathrm{c}}$ of the binary,

$$
D_{\mathrm{c}}:=D_{\mathrm{eff}}\left(\frac{\mathcal{M}_{1.4}}{\mathcal{M}}\right)^{5 / 6}
$$

For higher mass signals, the coalescence will occur within the sensitive band of the detector, as can be seen from equation (7). In this case, there is no simple relationship between the efficiency, chirp mass and distance, and both mass and effective distance must be taken into account when calculating the efficiency.

Let us now generalize to the case of several detectors. A given binary coalescence will have a different effective distance in each detector, and therefore the efficiency will be a function of the effective distance in each detector. In figure 3, we plot the efficiency against effective distance in the $\mathrm{H} 1$ and $\mathrm{L} 1$ detectors for a $1.4-1.4 M_{\odot}$ binary. At small effective distance in both detectors, the efficiency is unity, while at large distances it goes to zero. The shape of the constant efficiency contours depends upon two factors: the single instrument threshold and the combined threshold. At large effective distance in one instrument, but small 


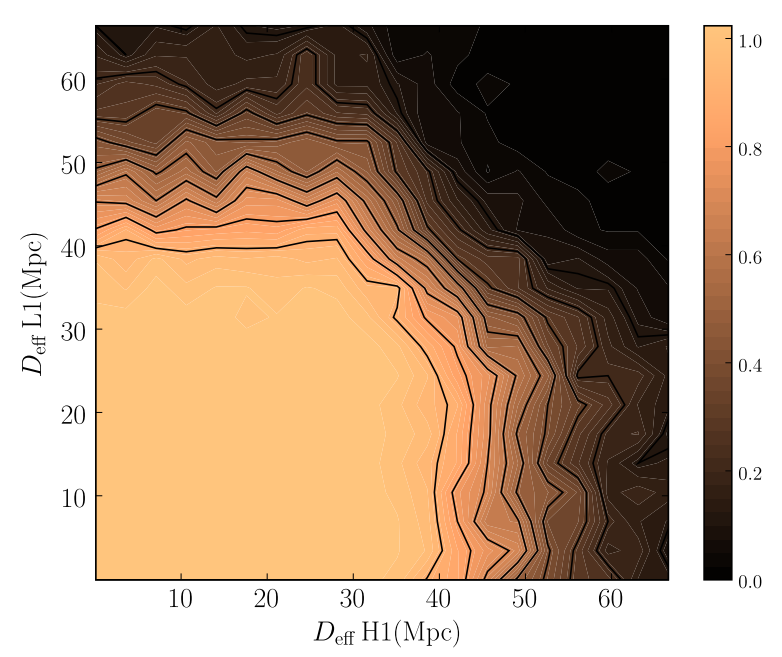

Figure 3. The detection efficiency as a function of the effective distance for the two detectors at Hanford (H1) and Livingston (L1). The efficiency at small distances is unity, while at large distances it is zero. The transition is governed by two effects. At large distances in one detector, the efficiency is limited by the single detector threshold of 5.5. At large distances in both instruments, the efficiency is determined by the combined SNR threshold of 10. The variations in the contours are due to the fact that a finite number of injections - 100 in each bin — were used when generating the efficiency curve.

in the other, the single instrument threshold limits the efficiency. For comparable distances, the efficiency is determined by the combined SNR.

3.2.2. Astrophysical model. In order to calculate the total luminosity to which a search is sensitive, we require the luminosity density as a function of the effective distance and mass. This involves combining a model of the location and luminosity of galaxies with the antenna response functions of the detectors, given in equation (2).

Let us consider a source from a given galaxy. The effective distance to the source depends upon the physical distance, and four sky angles - the sky location relative to the detector, the inclination and polarization angles. Equivalently, the location of the source can be described by $\vec{\lambda}=(D, \alpha, \delta, \iota, \psi, t)$, where the right ascension $\alpha$, declination $\delta$ and sidereal time $t$ serve to specify the source sky location. For sources from a particular galaxy, the distance, right ascension and declination of the galaxy $\left(D_{i}, \alpha_{i}, \delta_{i}\right)$ are known. Then, assuming that binary coalescences are uniformly distributed over the sidereal day, inclination and polarization angles, we obtain a distribution for the observed effective distances of sources from a given galaxy

$p_{i}\left(D_{\text {eff }}\right)=\int \mathrm{d} \vec{\lambda} p(t) p(\iota) p(\psi) \delta\left(D-D_{i}\right) \delta\left(\alpha-\alpha_{i}\right) \delta\left(\delta-\delta_{i}\right) \delta\left(D_{\text {eff }}-D_{\text {eff }}(\vec{\lambda})\right)$,

where $p(t)=1 /($ sidereal day), $p(\psi)=1 / 2 \pi$ and $p(\iota)=\sin (\iota) / 2$. In practice, this distribution is most easily obtained by simulating many signals, at random times and orientations, from a given galaxy.

In [8], the compact binary coalescence galaxy (CBCG) catalog has been compiled to a distance of $100 \mathrm{Mpc}$. For galaxies in this catalog the sky location and distance to the galaxy 


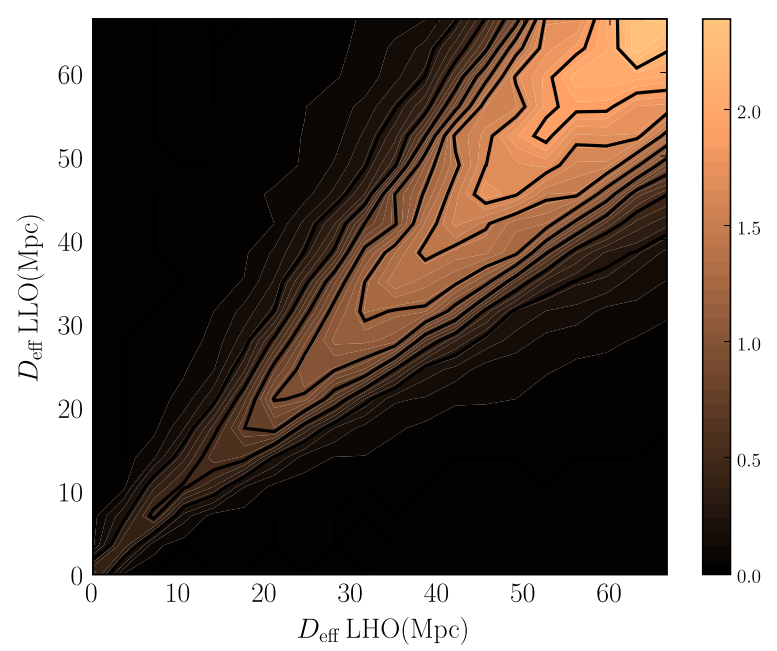

Figure 4. The luminosity distribution in the nearby universe as a function of the effective distance in the Hanford and Livingston detectors. The distribution is obtained by assuming that the binary coalescences in a given galaxy are dependent upon the blue-light luminosity of that galaxy and are uniformly distributed in sidereal time, inclination and polarization. The color bar indicates the available blue-light luminosity $\left(L_{10} / \mathrm{Mpc}^{2}\right)$. The number increases with distance in both detectors and is greatest on the diagonal. The off-diagonal spread in luminosity is due to the different alignments of the Hanford and Livingston detectors.

are known. In addition, the apparent magnitude $m_{B, i}$ in blue light of the galaxy is measured. The luminosity of the galaxy is obtained from its distance $D_{i}$ and apparent magnitude as

$$
\frac{L_{B, i}}{L_{B, \odot}}=\left(\frac{D_{i}}{10 \mathrm{pc}}\right)^{2} 10^{\left(M_{B, \odot}-m_{B, i}\right) / 2.5},
$$

where $L_{B, \odot}=2.16 \times 10^{33} \mathrm{ergs} \mathrm{s}^{-1}$ is the blue solar luminosity, and $M_{B, \odot}$ is the (absolute) blue solar magnitude [34].

Given the distribution of effective distances for each galaxy, it is straightforward to obtain the total luminosity as a function of effective distance by summing over all galaxies,

$$
L_{B}\left(D_{\text {eff }}\right)=\sum_{i} L_{B, i} p_{i}\left(D_{\text {eff }}\right)
$$

As before, this can be easily generalized to a distribution of luminosity as a function of effective distance for several detectors. In figure 4 we make use of the galaxy catalog of [8] to plot the luminosity as a function of the effective distance at the Hanford and Livingston detectors.

Finally, we must include the mass distribution $p(\mathcal{M})$. The available luminosity is expressed as a function of effective distance and chirp mass as

$$
L_{B}\left(D_{\mathrm{eff}}, \mathcal{M}\right)=\left(\sum_{i} L_{B, i} p_{i}\left(D_{\mathrm{eff}}\right)\right) p(\mathcal{M}) .
$$

For neutron star binaries, the mass distribution can be taken from observations [35] or, alternatively, from population synthesis [9]. For binaries containing at least one black hole, the lack of observations leads to greater uncertainty in the mass distribution, whence it is more natural to calculate a mass-dependent rate limit. 
3.2.3. Calculating the cumulative luminosity. The cumulative luminosity available to a search is obtained by multiplying the luminosity distribution in equation (30) against the efficiency $\epsilon\left(\rho, D_{\text {eff }}, \mathcal{M}\right)$ and integrating over the mass and effective distance,

$$
\mathcal{C}_{L}(\rho)=\int \mathrm{d} \mathbf{D}_{\text {eff }} d \mathcal{M} \epsilon\left(\rho, \mathbf{D}_{\text {eff }}, \mathcal{M}\right) L_{B}\left(\mathbf{D}_{\text {eff }}\right) p(\mathcal{M}) .
$$

For a given mass, this reduces to multiplying the data from figures 3 and 4 and integrating over the effective distances. This must then be integrated over the chirp mass to obtain the cumulative luminosity. For low-mass systems, the calculation is greatly simplified by recalling that the efficiency depends only upon the chirp distance (26) at a given site.

In a similar manner, the derivative of the cumulative luminosity $\mathcal{C}_{L}^{\prime}\left(\rho_{m}\right)$ can be calculated. This is done by calculating the rate of change of efficiency as a function of SNR, evaluated at $\rho_{m}$. With this information, we can calculate

$$
n_{F}=\frac{\left|\mathcal{C}_{L}^{\prime}\left(\rho_{m}\right)\right|}{\mathcal{C}_{L}\left(\rho_{m}\right)}
$$

In cases where the mass distribution is not known, the luminosity can be expressed as a function of the mass,

$$
\mathcal{C}_{L}(\rho, \mathcal{M})=\int \mathrm{d} \mathbf{D}_{\text {eff }} \epsilon\left(\rho, \mathbf{D}_{\text {eff }}, \mathcal{M}\right) L_{B}\left(\mathbf{D}_{\text {eff }}\right) .
$$

Then, the rate limit is naturally expressed as a function of the mass.

We can compute the $\mathcal{C}_{L}$ for the example with a loudest event SNR of 10. To simplify the calculation, assume that the mass of all binary components is $1.4 M_{\odot}$. Then, the total luminosity and its derivative are

$$
\mathcal{C}_{L}\left(\rho_{m}\right)=540 L_{10} \quad \text { and } \quad \mathcal{C}_{L}^{\prime}\left(\rho_{m}\right)=120 L_{10} .
$$

This gives a value of $n_{F}=0.22$. For comparison, we can calculate the expected value for a uniformly distributed population. In this case, $\mathcal{C}_{L} \propto \rho^{-3}$, whence $n_{F} \approx 3 / \rho_{m}$, which is similar to the calculated value. The difference is due to the fact that, at the distances under consideration, the non-uniformity of the luminosity distribution is important.

\subsection{Obtaining an upper limit}

In the preceding sections, we have described all of the pieces which are required in calculating an upper limit from a search for gravitational waves from binary coalescence. The calculation of the cumulative luminosity $\mathcal{C}_{L}$ depends upon the efficiency of the search, and the astrophysical distribution of signals. We have argued that, in the non-spinning case, these are both described as functions of effective distance and chirp mass. The quantity $\Lambda$ depends upon the foreground and background distributions, encoded in $n_{F}$ and $n_{B}$. The first of these, $n_{F}$ is obtained from the cumulative luminosity and its derivative, while $n_{B}$ is determined from analysis of events arising in analysis of time shifts of the data.

The formula for the upper limit was given in equation (21). Taking the prior distribution $p(R)$ of the rate to be uniform, we obtain a Bayesian upper limit with confidence $\alpha$ as

$$
1-\alpha=\mathrm{e}^{-R T \mathcal{C}_{L}\left(\rho_{m}\right)}\left[1+\left(\frac{\Lambda}{1+\Lambda}\right) R T \mathcal{C}_{L}\left(\rho_{m}\right)\right] .
$$

For a representative loudest event, we obtain $n_{B} \approx \rho_{m}$ and $n_{F} \approx 3 / \rho_{m}$. Therefore, $\Lambda \sim 3 / \rho_{m}^{2}$. For any realistic search, with a loudest event of SNR around 10, this implies that $\Lambda \ll 1$, and therefore the loudest event is most likely background. To obtain a mass-dependent upper limit, this analysis is repeated for different values of $\mathcal{M}$ making use of the mass-dependent 
luminosity function $\mathcal{C}_{L}\left(\rho_{m}, \mathcal{M}\right)$ to obtain a rate limit $R(\mathcal{M})$. This method neglects the fact that the distribution of background events is also mass dependent by using the same loudest event for all masses.

Plugging into the values obtained in the previous sections, we have $\Lambda=0.22 / 10.9=$ 0.020 , and $\mathcal{C}_{L}\left(\rho_{m}\right)=540 L_{10}$. Assuming a year of analysis time, the limit would be

$$
R_{90 \%}=\frac{2.35}{\mathcal{C}_{L}\left(\rho_{m}\right) T}=4.3 \times 10^{-3} L_{10}^{-1} \mathrm{yr}^{-1}
$$

This gives a reasonable estimate of the expected BNS upper limit in the absence of a detection in the LIGO S5 science run.

How does this compare with astrophysical predictions? The rate of galactic binary neutron star mergers is estimated to be $8.3_{-6.6}^{+20.9} \times 10^{-5} \mathrm{yr}^{-1}$, at $95 \%$ confidence [36]. Assuming the rate is indeed a function of the blue-light luminosity alone, and using $1.7 L_{10}$ as the blue-light luminosity of the Milky Way [36], gives an optimistic rate of $1.7 \times 10^{-4} L_{10}^{-1} \mathrm{yr}^{-1}$, which is a factor of 25 lower than the expected upper limit from the analysis of 1 year of data from initial LIGO.

\section{Systematic errors}

In the previous section, we have described a method for calculating an astrophysical upper limit or interval for the rate of binary coalescences from the results of a gravitational-wave search. The probability distribution for the rate is dependent on four quantities: the prior distribution $p(R)$, the cumulative luminosity $\mathcal{C}_{L}$, the relative probability $\Lambda$ of the loudest event being a signal, and the analysis time $T$. Of these quantities, only the analysis time can be unambiguously measured. The choice of prior distribution $p(R)$ will affect the posterior distribution. However, we take the prior as an input to the analysis and do not consider uncertainties associated with the choice of prior. There are numerous systematic errors which will affect the measured values of both the luminosity and the function $\Lambda$. These systematic effects can be broadly split into five categories:

- Imprecise knowledge of the astrophysical distributions of the mass and distance of binaries.

- Differences between the physical signal and the non-spinning, restricted post-Newtonian waveforms.

- Statistical fluctuations in the measured efficiency.

- Uncertainties in instrumental calibration.

- Errors in the calculated value of $\Lambda$, arising from the above uncertainties and errors in the background estimation.

In this section, we describe the various sources of uncertainty and analyze their effect. Additionally, we perform a marginalization over these uncertainties to produce the rate distribution.

\subsection{Uncertainties in population model}

The cumulative luminosity of a search will depend critically upon the astrophysical model used. In particular, the luminosity distribution is sensitive to both the location and luminosity of galaxies within the reach of the search. In addition, since the amplitude and frequency range of gravitational waves emitted by a binary coalescence is mass dependent, the cumulative luminosity will also be dependent upon the astrophysical mass distribution. In section 4.1.1, 
we discuss the systematics associated with uncertainties in galaxy distribution, while in 4.1.2 we investigate the effect of changing the mass population.

4.1.1. Galaxy distribution. The sky position of nearby galaxies is known accurately enough that errors in the right ascension and declination will not affect the cumulative luminosity. However, the distances and luminosity of galaxies are not so well known, whence these uncertainties must be taken into account when calculating the total luminosity. Indeed, the luminosity of a galaxy is not directly measurable, instead it is calculated from the apparent blue magnitude $m_{B, i}$ and distance $D_{i}$ using (28). The uncertainties in distances vary from less than $10 \%$, from observations of Cepheids in nearby galaxies observed with the Hubble Space Telescope, to uncertainties up to $30 \%$ for more distant galaxies. Additionally, there are uncertainties in the apparent magnitude of galaxies which vary from $\Delta m_{B, i}=0.3$ to $\Delta m_{B, i}=0.38[8]$.

We begin by considering an error in distance of $\Delta D_{i}$ to a galaxy at distance $D_{i}$. The change in distance of the galaxy will lead to a linear change in the effective distance of all sources from that galaxy. More precisely, changing the distance from $D_{i}$ to $\left(D_{i}+\Delta D_{i}\right)$ will change to distribution of effective distances from $p_{i}\left(D_{\text {eff }}\right)$ to $p_{\Delta, i}\left(D_{\text {eff }}\right)$ where

$$
p_{\Delta, i}\left[D_{\text {eff }}\left(1+\Delta D_{i} / D_{i}\right)\right]=p_{i}\left(D_{\text {eff }}\right) .
$$

Thus, the average effective distance to a source will increase by $\left(1+\Delta D_{i} / D_{i}\right)$. This will not have any effect for close (or very distant) galaxies where the efficiency to sources from that galaxy is unity (or zero). However, for galaxies at distances where the efficiency is rapidly changing, this can be a significant effect, reducing the efficiency when the distance to the galaxy is increased.

Since the luminosity of a galaxy is inferred from its measured distance and apparent magnitude, a change in the distance will also affect the calculated luminosity. It follows directly from (28) that a change in distance of $\Delta D_{i}$, leaving the apparent magnitude unchanged, will yield a change in luminosity $\Delta L_{B, i}$ given by

$$
\frac{L_{B, i}+\Delta L_{B, i}}{L_{B, i}}=\left(\frac{D_{i}+\Delta D_{i}}{D_{i}}\right)^{2} .
$$

Thus, the inferred luminosity will increase if the distance to the galaxy increases. This effect will be significant for all galaxies to which the search has nonzero efficiency.

It is also straightforward to calculate the effect of errors in the reported apparent magnitude. We have from equation (28)

$$
\frac{L_{B, i}+\Delta L_{B, i}}{L_{B, i}}=10^{\left(\Delta m_{B, i} / 2.5\right)} .
$$

Therefore, an increase (decrease) in magnitude will lead to an increase (decrease) in $\mathcal{C}_{L}$. The reported errors in the CBCG catalog are $\Delta m_{B, i}$ between 0.3 and 0.38 , giving corresponding uncertainties in the luminosity of $32 \%$ and $42 \%$ respectively.

In order to estimate the uncertainty in the luminosity $\mathcal{C}_{L}$, it is necessary to vary the distance and magnitudes of all galaxies for which the search has nonzero sensitivity. We expect that the errors are independent for different galaxies, although it is certainly conceivable that there is an overall systematic for a given galaxy catalog. However, to be conservative, we calculate the errors by moving all galaxies either closer or further. As with the distance error, we take the conservative error by increasing or decreasing the magnitude of all galaxies together.

At large distances, the luminosity distribution can be taken as homogeneous and isotropic. In this case, the blue luminosity density is calculated directly from observations 
as $L_{B}=(1.98 \pm 0.16) \times 10^{-2} L_{10} \mathrm{Mpc}^{-1}$ [8]. Thus, at distances greater than $40 \mathrm{Mpc}$, the uncertainty in luminosity can be calculated directly.

Recent papers have suggested that due to the large coalescence times for binary inspiral, a significant fraction of them might occur in elliptical galaxies where the star formation rate is low. The general loudest event formalism presented in [5] can be used in this case, and one might envision introducing two unknown rate parameters $R_{B}$ which depends upon the blue-light luminosity discussed above and $R_{E}$ which is a second contribution due to elliptical galaxies. Then, the rate would depend upon two parameters, and a given search could be used to place a confidence bound in the two-dimensional space spanned by them. If the corrections from including elliptical galaxies prove to be significant, this effect will be folded into future rate calculations.

4.1.2. Binary mass distribution. There is significant uncertainty in the mass distribution of coalescing binaries. Several binary neutron star systems, and significant numbers of single neutron stars, have been observed as pulsars, allowing us to place some restrictions on the mass distribution. However, the mass distributions presented in (for example) [9] are produced using large scale simulations which must assume an equation of state for the nuclear material. For stellar mass black hole binaries, there is little restriction on the mass distribution. These uncertainties lead us to place mass-dependent upper limits. However, it is still instructive to look at the sensitivity of our binary neutron star search for various mass distributions.

As discussed above, the distance to which a source can be observed is dependent upon its chirp mass. To leading order, the amplitude of the emitted gravitational radiation, and hence the distance to which the source can be observed, is proportional to $\mathcal{M}^{5 / 6}$. The astrophysical mass distribution of neutron stars can have a significant effect upon the distance to which sources are observable. For a $1.4-1.4 M_{\odot}$ solar mass binary $\left(\mathcal{M}=1.22 M_{\odot}\right)$, the $50 \%$ efficiency point for initial LIGO at SNR 7 occurs at $40 \mathrm{Mpc}$, whereas for a 3.0-3.0 $M_{\odot}$ binary that is increased to $75 \mathrm{Mpc}$. So, an astrophysical population containing higher mass binaries will increase our range. As an example, let us consider two mass distributions:

- The distribution of observed masses of radio pulsars [35], namely a Gaussian distribution peaked at $1.35 M_{\odot}$, with a width of $0.04 M_{\odot}$.

- The distribution from [9] obtained from population synthesis models. This is the mass distribution which was used in obtaining the LIGO S1 and S2 results given in $[37,38]$.

Figure 5 shows the distribution of chirp mass for the two different cases. In both cases, the peak is at around the same chirp mass of $1.2 M_{\odot}$, corresponding to binaries with component mass around $1.4 M_{\odot}$. However, there is a significant fraction of higher mass neutron stars in the population synthesis distribution.

Given a mass distribution, we can integrate the efficiency over the mass to obtain efficiency as a function of effective distance alone,

$$
\epsilon\left(D_{\text {eff }}\right)=\int \mathrm{d} \mathcal{M} p(\mathcal{M}) \epsilon\left(D_{\text {eff }}, \mathcal{M}\right)
$$

This curve is also plotted in figure 5 for the two mass distributions of interest. Varying the mass distribution has a $20 \%$ effect on the distance at which the efficiency reaches $50 \%$. Since the choice of mass distribution can have a significant effect on the efficiency, in [10] the upper limits from LIGO searches are reported for a stated sample distribution. The mass uncertainty is not folded into the systematic errors on the upper limit. 

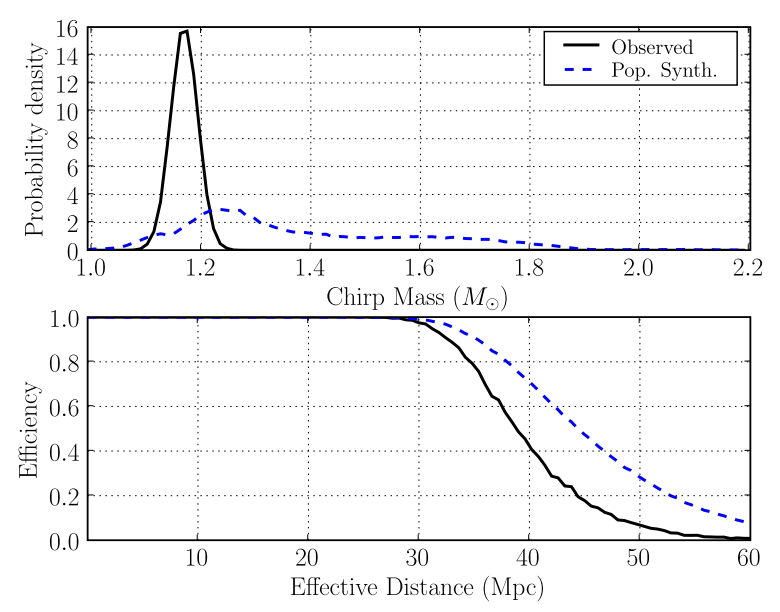

Figure 5. Effect of the mass distribution on the search efficiency. The upper plot shows two different binary neutron star mass populations: one taken directly from observed neutron star masses, which are fitted to a Gaussian mass with a peak at $1.35 M_{\odot}$ and width of $0.04 M_{\odot}$; the other from population synthesis models in [9]. The lower plot shows the efficiency as a function of effective distance for the initial LIGO detector at SNR 7, where mass has been folded in.

\subsection{Uncertainties in the waveform}

In several places, we have assumed that both the physical waveforms and the search templates are described by the post-Newtonian approximation and we have ignored the effects of spin. The methods presented here are not tied to the particular templates used and extend immediately to searches using other types of waveform, or even to excess-power-type analyses for unmodeled waveforms [12]. The only requirement is that it is possible to meaningfully associate an SNR to both noise and simulated signals, and hence obtain a loudest event. Throughout, we have also modeled the true astrophysical waveforms as those obtained from the restricted, non-spinning, post-Newtonian calculation. This can have a significant effect on the interpretation of the result.

In order to explore how the uncertainty in the simulated waveforms will affect our result, it is useful to introduce the notion of match. For a given set of parameters (in particular the component masses and spins), we denote the true waveform by $h_{t}$ and those used in simulations as $h_{s}$. The difference between the true and simulated waveforms is encoded in the match $M$ defined as

$$
M=\frac{\left(h_{t} \mid h_{s}\right)}{\sqrt{\left(h_{t} \mid h_{t}\right)\left(h_{s} \mid h_{s}\right)}} .
$$

If the waveforms agree perfectly, the match will be unity, while in all other cases it will be less than one.

Differences between the post-Newtonian approximation and the true waveforms have been examined in some detail. In [39, 40], this has been done by comparing the postNewtonian waveforms to those obtained from black hole perturbation theory. The results indicate approximately a $10 \%$ loss of SNR (i.e. a match of $90 \%$ ) due to inaccurate modeling of the waveforms for low-mass systems, with the effect becoming more pronounced for higher mass ratios. In [41] a similar result was obtained by comparing waveforms at different postNewtonian order. Recent breakthroughs in numerical simulations of black hole and neutron star coalescences will allow for further exploration of this issue. 
In order to fully address the issue of spin, it will be necessary to perform substantial Monte Carlo simulations of spinning waveforms. For the time being, we rely on estimates provided by Apostolatos [23] in which he shows that less than $10 \%$ of all spin-orientations and parameters consistent with binary neutron stars provide a loss of SNR greater than 5\%.

In BNS searches, where the same waveforms are used as templates and simulated signals, it is straightforward to calculate the effect of any mismatch. In this case, the mismatch between the astrophysical waveform and the post-Newtonian approximation will lead to an over-estimation of the observed SNR from a given binary coalescence. Specifically, if $\rho_{t}$ and $\rho_{s}$ are the SNRs associated with the true signal and simulation respectively, then

$$
\rho_{t}=\rho_{s} M \sqrt{\frac{\left(h_{t} \mid h_{t}\right)}{\left(h_{s} \mid h_{s}\right)}} .
$$

Therefore, the SNR associated with a true signal is reduced by a factor $M$ from what is observed in a simulation.

There is an important difference between the waveform uncertainties and the other systematic errors discussed in this section. In the simulation, we are using the same waveform for injection and detection. In reality, the true astrophysical waveforms will not match precisely the detection family. This will lead to a decrease in the overlap between the astrophysical and detection families. It is not possible for this to lead to an increase as the match cannot be greater than unity. Thus, the waveform errors can only serve to decrease the cumulative luminosity available to a search. However, in cases where the simulated signals and templates do not match precisely, it is possible that the 'true' waveforms will have a better match with the templates than the simulations do. Then, errors in the waveform may cause us to underestimate the efficiency of the search.

Returning to equation (42), we see that the overall normalization of the waveform will also affect the SNR. Generally, it is assumed that the amplitude of the simulated and astrophysical waveforms are in good agreement, namely $\left(h_{s} \mid h_{s}\right) \approx\left(h_{t} \mid h_{t}\right)$. In [42, 43], the authors consider the effects of higher order post-Newtonian corrections to the amplitude. These are particularly important for higher masses, especially when the ratio of the component masses is large. Furthermore, in [42] it has been noted that the inclusion of amplitude corrections actually decreases the overall amplitude of the signal. Thus, even though neglecting amplitude corrections may not significantly affect the detection ability of a search, it can still have an effect on the interpretation of results. This effect is not important for BNS systems, but does become important in higher mass, asymmetric systems.

\subsection{Uncertainties in the instrumental calibration}

When performing simulations of the gravitational-wave signal from a coalescing binary, the SNR $\rho_{s}$ associated with a simulated signal differs from the SNR $\rho_{t}$ that would be associated with a real signal with the same parameters due to uncertainties in the instrumental calibration.

In calculating the efficiency of a search, simulated events are added in software to the data after it has been recorded. Therefore, any uncertainty in instrumental calibration will not affect the software injections in the way it will a real signal. To quantify this effect, we focus on the differences between a true signal with given masses and effective distance $D_{\text {eff }}$ and a simulated signal with the same parameters. To simplify matters, we assume that the waveform exactly matches one of the search templates (i.e. ignore the waveform uncertainty discussed above), in which case

$$
\tilde{s}(f)=\frac{\mathrm{e}^{\mathrm{i} \phi_{0}}}{D_{\text {eff }}} \tilde{h}_{c}(f)+\tilde{n}(f),
$$


where $\tilde{h}_{c}(f)$ is the waveform introduced in $(6), \phi_{0}$ is an arbitrary phase, and $n(f)$ is the detector noise. Then, by substitution into equation (12), it is straightforward to verify that $\left\langle\rho^{2}\right\rangle=\rho_{h}^{2}+2$ as expected.

The output of a gravitational-wave detector is not the gravitational wave strain, it is an uncalibrated signal $v(t)$. This output is related to the strain by

$$
\tilde{s}(f)=R_{t}(f) \tilde{v}(f),
$$

where $R_{t}(f)$ is the true response function of the instrument. The process of calibrating the data involves the construction of a response function $R(f)$. Due to calibration uncertainties, the reconstructed response will differ from the true response. These calibration uncertainties can, to some degree, be independently tested by performing 'hardware injections' into the detectors [44]. To calculate the effect of calibration uncertainties, let us follow [45] and write the measured response as

$$
R(f)=r(f) \mathrm{e}^{\mathrm{i} \theta(f)}
$$

and the true response as

$$
R_{t}(f)=[r(f)+\delta r(f)] \mathrm{e}^{\mathrm{i}[\theta(f)+\delta \theta(f)]},
$$

where $\delta r$ and $\delta \theta$ are the amplitude and phase parts of the calibration error which are assumed to be small.

In the event that a gravitational wave with strain $h(t)$ impinges on the detector, the calibrated strain reconstructed from the output of the detector will be given by ${ }^{5}$

$$
\tilde{s}_{t}(f)=\frac{R(f)}{R_{t}(f)} \frac{\mathrm{e}^{\mathrm{i} \phi_{0}} \tilde{h_{c}}(f)}{D_{\text {eff }}} .
$$

The SNR for an event observed in the detector can be calculated by substituting equation (47) into the expression (12) for the SNR. Then, making use of the expressions (45) and (46) for the response functions, and expanding in powers of $\delta r / r$ and $\delta \theta$ we obtain

$\rho_{t}^{2}=\rho_{h}^{2}-\frac{\rho_{h}^{2}}{\sigma^{2}}\left[4 \int_{0}^{\infty} \mathrm{d} f \frac{\left|\tilde{h}_{c}(f)\right|^{2}}{S_{n}(f)}\left(\frac{2 \delta r}{r}+(\delta \theta)^{2}\right)\right]+\frac{\rho_{h}^{2}}{\sigma^{4}}\left(4 \int_{0}^{\infty} \mathrm{d} f \frac{\left|\tilde{h}_{c}(f)\right|^{2}}{S_{n}(f)} \delta \theta\right)^{2}$.

Thus, the error is linear in $\delta r$ but quadratic in $\delta \theta$. Furthermore, it follows directly from the Schwarz inequality that

$$
\left|\left(\tilde{h}_{c} \delta \theta \mid \tilde{h}_{c}\right)\right|^{2} \leqslant \sigma^{2}\left(\tilde{h}_{c} \delta \theta \mid \tilde{h}_{c} \delta \theta\right)
$$

This guarantees that the magnitude of the second (positive) contribution to the SNR from the phase error is always less than the first (negative) one. Hence, an error in the phase of the response function will always serve to reduce the recovered SNR.

4.3.1. Application to the LIGO instruments. The calibration report from the LIGO S4 science run [46] provides an in depth analysis of the calibration of the LIGO instruments. Here, we briefly review those details which are relevant for coalescing binary searches.

In the LIGO instruments, the response function is determined from the sensing function $C(f)$ and the open-loop gain function $G(f)$ as

$$
R(f)=\frac{1+G(f)}{C(f)}
$$

5 For simplicity, we ignore contributions from the noise in the following. 
These calibration functions, $C(f)$ and $G(f)$ are measured at intervals during an analysis. At intervening times, it is assumed that their functional form is unchanged. However, due to the changes in light power stored in the arms, a time-dependent rescaling $\gamma(t)$ of the both the sensing function and open-loop gain is required. Thus, the response function for any time can be expressed as

$$
R(f, t)=\left(\frac{1+\gamma(t) G_{o}(f)}{\gamma(t) C_{o}(f)}\right)
$$

The uncertainties in the various components of the response function are calculated in detail in [46]. These can be summarized by the statement that the uncertainties in the response function are of order $5 \%$ in the amplitude of the response function and $5^{\circ}$ in phase. Substituting this into our general expression (48), we obtain

$$
\rho_{t}=\rho\left[1 \mp \frac{|\delta r|}{r}-\frac{|\delta \theta|^{2}}{2}\right]=\rho[1 \mp 0.05] .
$$

Note that the error in the measured SNR is primarily due to the amplitude calibration uncertainty.

\subsection{Uncertainties in the measured efficiency}

The effects of discreteness of the template placement, errors in the estimates of the power spectral density $S(f)$ used in the matched filter in equation (12), and trends in the instrumental noise are all accounted for by the Monte Carlo simulation. However, in the Monte Carlo simulation, only a finite number of injections are performed as these are computationally costly. Thus, the efficiency plots, such as that shown in figure 2, have an associated statistical error. The efficiency plots are produced by binning up the parameter space and calculating the efficiency in each bin as

$$
\epsilon=\frac{N_{f}}{N_{f}+N_{m}},
$$

where $N_{f}$ and $N_{m}$ are the number of found and missed injections respectively. Then, assuming binomial errors for the efficiency, the variance in the efficiency is

$$
\sigma_{\epsilon}^{2}=\frac{N_{f} N_{m}}{\left(N_{f}+N_{m}\right)^{3}}=\frac{\epsilon(1-\epsilon)}{\left(N_{f}+N_{m}\right)} .
$$

As expected, the variance is inversely proportional to the number of injections performed. Furthermore, it is clear from (54) that the statistical uncertainty in the efficiency is greatest when the efficiency is close to $50 \%$. The Monte Carlo error in the luminosity is obtained by multiplying the error in the efficiency by the luminosity ${ }^{6}$,

$$
\left(\Delta \mathcal{C}_{L}(\rho)\right)^{2}=\sum_{D_{\mathrm{eff}}, \mathcal{M}} \sigma_{\epsilon}^{2}\left(\rho, \mathbf{D}_{\mathrm{eff}}, \mathcal{M}\right)\left[L_{B}\left(\mathbf{D}_{\mathrm{eff}}\right) p(\mathcal{M})\right]^{2}
$$

When performing a search on real data, software injections are computationally costly. From equation (55), we see that simulations are most efficiently performed when concentrated where the efficiency is close to $50 \%$ and there is a significant contribution to the luminosity.

\footnotetext{
6 Strictly, there is a second Monte Carlo uncertainty due to errors in the calculated luminosity. However, the luminosity density of figure 4 can be calculated to good accuracy with minimal computational cost so that these errors will be insignificant.
} 
Furthermore, for low-mass signals, making use of the chirp distance (26) reduces the dimension of (55) by one and consequently reduces the size of the associated Monte Carlo errors.

\subsection{Marginalization over uncertainties to obtain an upper limit}

In section 3 we have described how to calculate an upper limit from an inspiral search, making use of the loudest event statistic. This involves calculating three quantities, the amount of time searched $T$, the total luminosity $\mathcal{C}_{L}\left(\rho_{m}\right)$ to which the search is sensitive, and $\Lambda$. In this section, we have discussed various systematic errors which affect our ability to measure these quantities. Here, we will describe how these uncertainties can be marginalized over to produce an upper limit which takes them into account.

Let us begin by considering a single uncertainty (for example the distance error) which will effect the luminosity $\mathcal{C}_{L}$. Then, by evaluating errors associated with this uncertainty, we obtain a probability distribution for the cumulative luminosity, $p_{d}\left(\mathcal{C}_{L}\right)$. In order to marginalize over the distance uncertainty, we simply evaluate

$$
p\left(R \mid \rho_{m}, T, B\right)=\int \mathrm{d} \mathcal{C}_{L} p_{d}\left(\mathcal{C}_{L}\right) p\left(R \mid \rho_{m}, T, B, \mathcal{C}_{L}\right),
$$

where the probability distributions on the right-hand side must be normalized to unity. This is straightforward when there is only one error to take into account. However, in the preceding sections, we have detailed several errors. It is not practical to integrate over all of these errors independently, so we perform a Monte Carlo integration. For the majority of the errors, we use a Gaussian with standard deviation given by the value stated above (and truncated so that the total $L_{10}$ will never be negative). Since the magnitude error affects the luminosity exponentially, assuming a Gaussian error on this leads to a log-normal distribution for the luminosity. Finally, for the waveform error, we use a one-sided Gaussian, i.e. one which can only decrease the cumulative luminosity. Then, the Monte Carlo integral is performed by sampling many times from the appropriate distributions.

\subsection{Uncertainties in $\Lambda$}

We have cataloged various uncertainties which will affect the calculated luminosity $\mathcal{C}_{L}$ for a given search and shown how they can be marginalized over to obtain a final distribution for the rate. In addition, we need to examine the effect any uncertainty, in the estimation of the parameter $\Lambda$, will have upon the upper limit. To do so, we will once again marginalize over this nuisance parameter (as in equation (56)), to obtain a distribution which is independent of $\Lambda$. However, due to the simple manner in which $\Lambda$ enters the probability distribution (17) for the rate, to leading order the marginalization procedure has no effect on the distribution [5]. More precisely, provided the uncertainties in $\Lambda$ are small compared to $(1+\Lambda)$, the marginalization procedure serves to replace $\Lambda$ by its expectation value. We have argued that for a typical search where the loudest event is consistent with the background, we expect $\Lambda \ll 1$ whence it is safe to neglect uncertainties in its value.

There are cases when the estimated background from time shifts will not accurately reflect the background. An obvious example of this is when computing the background for the colocated Hanford detectors. It is well known that there are correlated noise sources which will produce inspiral triggers in the two instruments simultaneously. While every effort is made to remove these times by examination of auxiliary channels, and in particular the seismic data, there are still invariably some correlated noise events which occur in the Hanford data. In this case, time shifts will not give a good estimate of the background. There are other ways to estimate the background, such as using 'reverse chirp' filters (obtained by time inverting the 
template). However, in calculating an upper limit, an underestimation of the background will lead to a conservative upper limit being placed.

\subsection{An example}

To illustrate the issues associated with these systematic uncertainties, we will calculate them for the example introduced earlier. For our example, the simulated loudest event had a combined SNR of $\rho_{m}=9.95$, which corresponds to a single instrument SNR around 7. At this SNR, the $50 \%$ efficiency for BNS occurs at $40 \mathrm{Mpc}$. The cumulative luminosity of such a search is $\mathcal{C}_{L}\left(\rho_{m}\right)=540 L_{10}$. Finally, the value of $\Lambda$ for our simulated results is 0.02 . Now, we turn our attention to the systematic uncertainties discussed above. We will evaluate the effect of each of these on the cumulative luminosity.

Distance uncertainties are obtained by moving all galaxies either closer or further away, by the appropriate fraction given in the CBCG-catalog. This changes the effective distance to sources according to equation (37) and the luminosity according to equation (38). This yields a change in cumulative luminosity of $90 L_{10}$ with a luminosity decrease as galaxy distances are increased. Uncertainties in the magnitudes of galaxies are taken into account by rescaling their luminosities according to equation (39) and lead to an error in the cumulative luminosity of $100 L_{10}$.

Based on the discussion of section 4.2, for BNS systems we choose to use a $10 \%$ uncertainty in the astrophysical waveform. This is simulated by reducing the efficiency of both detectors by the amount. In other words, we rescale the axes in figure 3 downwards by $10 \%$. The effect in our simulation is a change in the luminosity of $160 L_{10}$.

The calibration uncertainty is calculated by varying the efficiency curve accordingly, in a similar manner to that used for the waveform errors. Since the calibration errors in different instruments are independent, we consider them one at a time. In our example, we take a 5\% calibration uncertainty in both the $\mathrm{H} 1$ and $\mathrm{L} 1$ detectors. This is calculated to lead to a $37 L_{10}$ variation in the luminosity in $\mathrm{H} 1$ and a $34 L_{10}$ variation in $\mathrm{L} 1$. These numbers are very similar as expected. They differ slightly due to the fact that certain galaxies are better aligned for one detector than the other.

The Monte Carlo uncertainty can be calculated according to equation (55). As an example, figure 2 was produced with 100 injections in each bin. This gives a Monte Carlo error of $3 L_{10}$ which is already well below the errors due to astrophysical and waveform uncertainties.

The probability distribution for the cumulative luminosity taking into account these systematic errors is shown in figure 6. To generate the distribution, the uncertainties described above are used as the standard deviation of Gaussian distributions sampled via a Monte Carlo process to obtain the result. As is clear from the figure, the width of the probability distribution is significant. The greatest contributions to the uncertainty come from the astrophysicsthe uncertainty in the luminosity distribution of nearby galaxies - and uncertainties in the waveform. The systematic effects due to instrumental calibration and Monte Carlo errors are sub-dominant.

It is illustrative to consider the magnitude error and the waveform error in more detail. Although these errors are similar in magnitude, the waveform error is one sided, and thus has a more significant effect on the result. Although the magnitude error is $100 L_{10}$, marginalizing over it only increases the $90 \%$ confidence upper limit from $4.3 \times 10^{-3} L_{10}^{-1} \mathrm{yr}^{-1}$ to $4.5 \times 10^{-3} L_{10}^{-1} \mathrm{yr}^{-1}$. In contrast, marginalizing over only the waveform error increases the upper limit to $6.2 \times 10^{-3} L_{10}^{-1} \mathrm{yr}^{-1}$. The effect is much more significant, even though the magnitude of the two systematic errors is similar. The reason is that the waveform error can only decrease the sensitivity of the search. Indeed, by modeling the waveform error as a 


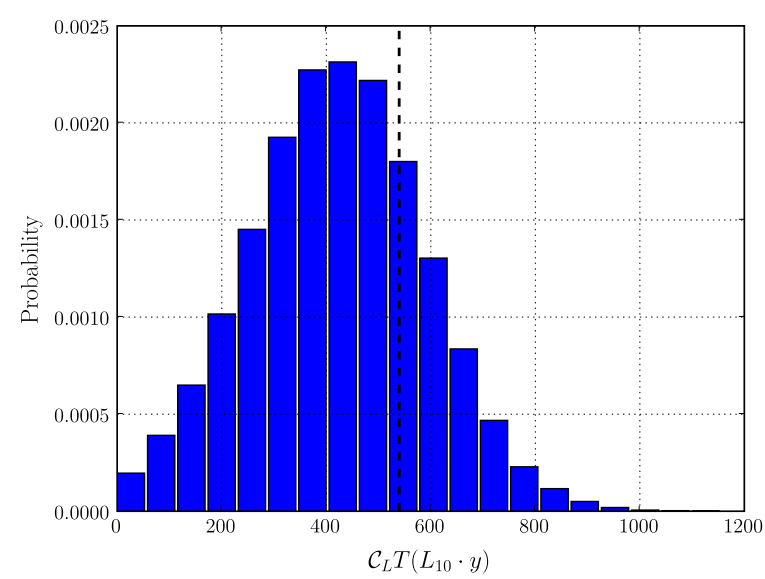

Figure 6. The distribution of the cumulative luminosity after marginalizing over the systematic errors. The histogram was produced by using 100,000 samples, and includes all the sources of error described in this section. The dashed vertical line gives the luminosity before marginalization. The fact that the peak of the distribution is below this is due to the fact that the waveform errors are one sided.

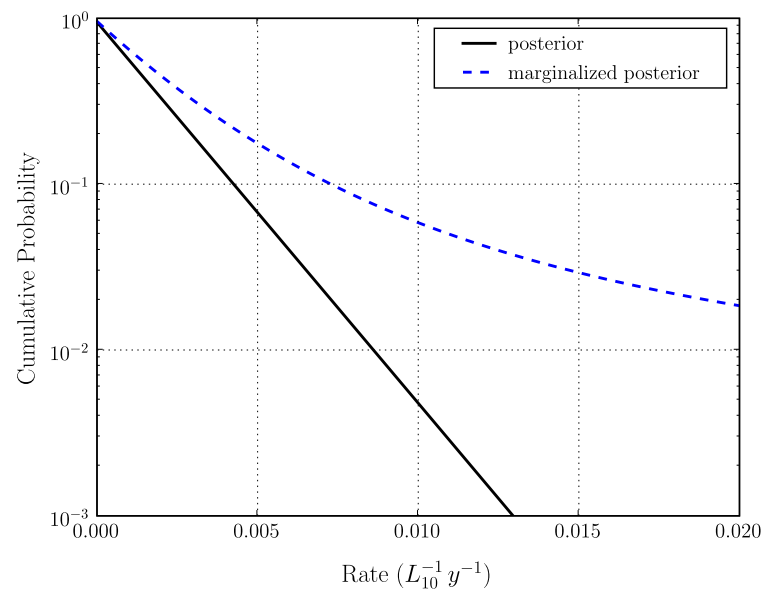

Figure 7. The cumulative distribution on the rate being greater than a given value. We are interested in the $90 \%$ upper limit and therefore take the rate for which the distribution is equal to 0.1 . The distribution is plotted for both the original luminosity of $540 L_{10}$ (solid line) and the marginalized luminosity distribution shown in figure 6 (dashed line). The large tail on the marginalized rate is due to the width of the luminosity distribution.

one-sided Gaussian, we are significantly changing the mean value of $\mathcal{C}_{L}\left(\rho_{m}\right)$, reducing it from $540 L_{10}$ to $410 L_{10}$, which accounts for a large fraction of the increase in the reported upper limit. When we include all of the systematics together, we obtain a luminosity distribution as shown in figure 6.

Finally, we can make use of the above distribution for the cumulative luminosity in order to construct the posterior probability distribution for the rate. We do this both for the unmarginalized and marginalized cases, and these are shown in figure 7 . This shows the 
cumulative posterior probability distribution for the rate of binary coalescence given the search. The figure shows both the un-marginalized and marginalized distributions. The marginalized distribution takes into account the systematic errors discussed above and falls off more slowly due to the width of the luminosity distribution from figure 6. The final, marginalized upper limit for this example can be read off from figure 7 as $7.3 \times 10^{-3} L_{10}^{-1} \mathrm{yr}^{-1}$.

\section{Summary and conclusions}

The astrophysical interpretation of the results is a critical part of any search for gravitational waves. In this paper, we have described a method to obtain an astrophysical rate upper limit or interval from the results of a search for coalescing binaries. This method has been used in obtaining the results from the LIGO S3 and S4 science runs [10]. An astrophysical interpretation of a search result requires a good understanding of both the detector sensitivity and the relevant astrophysics. We have argued that the detector sensitivity can be expressed in terms of the efficiency and furthermore that, for non-spinning systems, this efficiency is dependent only on the chirp mass and effective distance of the binary relative to the detectors. Furthermore, for low-mass systems, these can be combined into a single quantity, the chirp distance (26), which characterizes the amplitude. The use of effective distance is only appropriate for non-spinning binaries. Once spin is included, the orbital plane of the binary precesses whence the effective distance is not a constant over the course of inspiral. Despite this, the methods presented here could be extended to spinning binaries by making use of the 'expected SNR' ( $\rho_{h}$ in equation (14)) to characterize the amplitude of the signal. However, while it is clear that the efficiency for a non-spinning binary will be a function only of the effective distance and chirp mass, it is not obvious that the expected SNR and masses will completely characterize the efficiency to spinning systems.

The relevant astrophysics is encoded in the expected distribution of coalescing binaries in the universe. Following [6], we make the assumption that compact binaries are distributed according to the blue-light luminosity. Making use of a catalog of nearby galaxies (such as the one in [8]), we obtained an expression for the total luminosity to which a given search is sensitive. The loudest event statistic allows us to obtain a probability distribution for the rate of binary coalescence given the results of a search. This distribution depends upon the cumulative luminosity discussed above as well as an understanding of the rate of background, noise events present in the data. Finally, the posterior distribution for the rate can be used to calculate an upper limit or rate interval for the occurrence of binary coalescence.

There are numerous systematic uncertainties involved in the calculation of the rate which we have discussed in detail. The dominant errors arise due to uncertainties in the distribution of nearby galaxies and imprecise knowledge of the gravitational waveform emitted by coalescing binaries. It is reassuring that the errors associated with our understanding of the detectors and the analysis, such as calibration and Monte Carlo statistical uncertainties, are less significant than the physical and astrophysical uncertainties discussed above.

Uncertainties in the measurement of distances and apparent magnitudes of nearby galaxies leads to an uncertainty in the total luminosity available to a search. This in turn affects the reported rate limit. Additionally, the unknown mass distribution of coalescing binaries will have a significant effect on the reported rate. To mitigate this effect, rates are reported as a function of mass. In the future, gravitational-wave observations of binaries by advanced detectors will provide improved knowledge of both the mass and location distribution of binaries. Although the post-Newtonian waveform is known to a high level of accuracy, uncertainties in the waveform, the neglect of spin and amplitude corrections lead to significant uncertainties in the sensitivity of the search and hence the reported rate. In future searches, it 
should be possible to quantify the waveform uncertainties more precisely by performing simulations with amplitude corrected, spinning waveforms and by comparing the postNewtonian waveforms to those obtained from numerical relativity simulations.

\section{Acknowledgments}

We would like to acknowledge many useful discussions with members of the LIGO Scientific Collaboration inspiral analysis group which were critical in the formulation of the methods and results described in this paper. This work has been supported in part by NSF grant PHY-0200852, a Cottrell Scholar Award from the Research Corporation (PRB), and the Royal Society (SF). LIGO was constructed by the California Institute of Technology and Massachusetts Institute of Technology with funding from the National Science Foundation and operates under cooperative agreement PHY-0107417. This paper has LIGO document no LIGO-P070077-00-Z.

\section{References}

[1] Waldman S J (LIGO Scientific Collaboration) 2006 Class. Quantum Grav. 23 S653-60

[2] Acernese F et al 2006 Class. Quantum Grav. 23 S635-42

[3] Hild S 2006 Class. Quantum Grav. 23 S643-51

[4] Brady P R, Creighton J D E and Wiseman A G 2004 Class. Quantum Grav. 21 S1775-81

[5] Biswas R, Brady P R, Creighton J D E and Fairhurst S 2007 Preprint 0710.0465

[6] Phinney E S 1991 Astrophys. J. 380 L17

[7] Nutzman P, Kalogera V, Finn L S, Hendrickson C and Belczynski K 2004 Astrophys. J. 612 364-74

[8] Kopparapu R K et al 2008 Astrophys. J. 675 1459-67

[9] Belczynski K, Kalogera V and Bulik T 2002 Astrophys. J. 572407

[10] Abbott B et al (LIGO Scientific Collaboration) 2008 Phys. Rev. D 77062002

[11] Cutler C et al 1993 Phys. Rev. Lett. $702984-7$

[12] Anderson W G, Brady P R, Creighton J D E and Flanagan E E 2001 Phys. Rev. D 63042003

[13] Blanchet L, Damour T, Iyer B R, Will C M and Wiseman A G 1995 Phys. Rev. Lett. 74 3515-8

[14] Blanchet L, Damour T and Iyer B R 1995 Phys. Rev. D 515360

[15] Blanchet L, Iyer B R, Will C M and Wiseman A G 1996 Class. Quantum Grav. 13575

[16] Blanchet L, Faye G, Iyer B R and Joguet B 2002 Phys. Rev. D 65061501

[17] Blanchet L, Damour T, Esposito-Farèse G and Iyer B R 2004 Phys. Rev. Lett. 93091101

[18] Damour T, Iyer B R and Sathyaprakash B S 1998 Phys. Rev. D 57885

[19] Buonanno A and Damour T 1999 Phys. Rev. D 59084006

[20] Buonanno A and Damour T 2000 Phys. Rev. D 62064015

[21] Damour T, Iyer B R and Sathyaprakash B S 2001 Phys. Rev. D 63044023

[22] Apostolatos T A, Cutler C, Sussman G J and Thorne K S 1994 Phys. Rev. D 496274

[23] Apostolatos T A 1995 Phys. Rev. D 52605

[24] Brown D A 2004 Search for gravitational radiation from black hole MACHOs in the galactic halo PhD Thesis University of Wisconsin-Milwaukee

[25] Allen B A, Anderson W G, Brady P R, Brown D A and Creighton J D E 2005 Findchirp: an algorithm for detection of gravitational waves from inspiraling compact binaries Preprint gr-qc/0509116

[26] Thorne K S 1987 Gravitational radiation Three Hundred Years of Gravitation ed S W Hawking and W Israel (Cambridge: Cambridge University Press) chapter 9, pp 330-458

[27] Droz S, Knapp D J, Poisson E and Owen B J 1999 Phys. Rev. D 59124016

[28] Wainstein L A and Zubakov V D 1962 Extraction of Signals From Noise (Englewood Cliffs, NJ: Prentice-Hall)

[29] Babak S, Balasubramanian, Churches D, Cokelaer T and Sathyaprakash B S 2006 Class. Quantum Grav. 23 5477-504

[30] Abbott B et al (LIGO Scientific Collaboration) 2007 Tuning matched filter searches for compact binary coalescence Technical Report LIGO-T070109-01, LIGO Project

[31] Allen B 2005 Phys. Rev. D 71062001

[32] Rodríguez A 2007 Reducing false alarms in searches for gravitational waves from coalescing binary systems Master's Thesis Louisiana State University 
[33] de Freitas Pacheco J A , Regimbau T, Vincent S and Spallicci A 2006 Int. J. Mod. Phys. D 15 235-50

[34] Binney J and Tremaine S 2000 Galactic Dynamics (Princeton Ser. Astrophys.) (Princeton, NJ: Princeton University Press)

[35] Thorsett S E and Chakrabarty D 1999 Astrophys. J. 512288

[36] Kalogera V et al 2004 Astrophys. J. Lett. 614 L137-8

Kalogera V et al 2004 The cosmic coalescence rates for double neutron star binaries Astrophys. Phys. J. 601 L179 (erratum)

[37] Abbott B et al (LIGO Scientific Collaboration) 2004 Phys. Rev. D 69122001

[38] Abbott B et al (LIGO Scientific Collaboration) 2005 Phys. Rev. D 72082001

[39] Droz S and Poisson E 1997 Phys. Rev. D 564449

[40] Droz S 1999 Phys. Rev. D 59064030

[41] Canitrot P 2001 Phys. Rev. D 63082005

[42] Van Den Broeck C 2006 Class. Quantum Grav. 23 L51-8

[43] Van Den Broeck C and Sengupta A S 2007 Class. Quantum Grav. 24 155-76

[44] Brown D A (LIGO Scientific Collaboration) 2004 Class. Quantum Grav. 21 S797-800

[45] Allen B 1996 LIGO calibration accuracy Technical Report LIGO-T960189-00-Z, LIGO Project

[46] Dietz A, Garofoli J, González G, Landry M, O'Reilly B and Sung M 2006 Calibration of the LIGO detectors for S4 Technical Report LIGO-T050262-01-D, LIGO Project 